\title{
12
}

\section{High-Efficiency Power Amplifiers}

\author{
Guillermo Velasco-Quesada, Herminio \\ Martínez-García, and Alfonso Conesa-Roca
}

\section{CONTENTS}

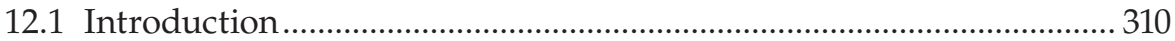

12.2 Fundamentals of Power Amplifiers and Output Stages ....................... 310

12.2.1 Concepts of Efficiency and Distortion for

a Power Amplifier

12.2.1.1 Efficiency in Power Amplifiers ........................................ 311

12.2.1.2 Distortion in Power Amplifiers........................................ 311

12.2.2 Power Amplifiers Classification..................................................... 312

12.2.3 Class-A Output Stages ................................................................... 314

12.2.4 Class-B Output Stages .................................................................... 315

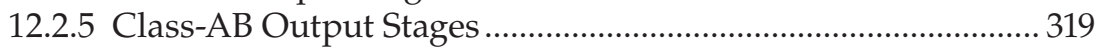

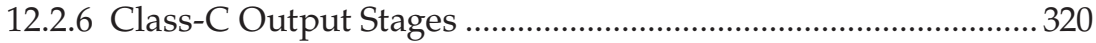

12.3 High-Efficiency Power Amplifiers and Output Stages........................... 324

12.3.1 Introduction and Context ................................................................324

12.3.2 Switching Amplifiers: Class-D Amplifiers...................................325

12.3.2.1 Half-Bridge Output Stage ...............................................328

12.3.2.2 Full-Bridge Output Stage ………………………….........328

12.3.3 Switching Amplifiers: Class-E Amplifiers ..................................329

12.3.4 Switching Amplifiers: Class-F Amplifiers....................................330

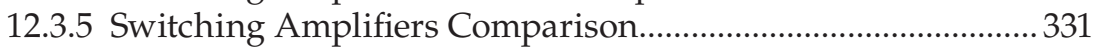

12.3.6 Amplifiers with Adjustable Power Supply Voltage......................332

12.3.6.1 Class-G Amplifiers............................................................332

12.3.6.2 Class-H Amplifiers .............................................................335

12.3.7 Other High-Efficiency Power Amplifier Topologies....................336

12.3.7.1 Class-FE and class-E/F Amplifiers..................................337

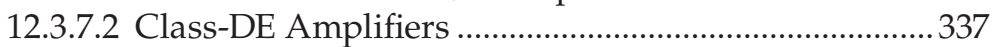

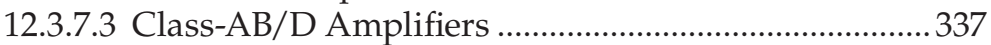

12.3.7.4 Class-DG Amplifiers.....................................................338

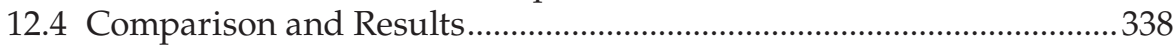

12.5 Would You like to Learn More? ..................................................................341

12.5.1 On Fundamentals of Power Amplifiers and Output Stages....341

12.5.2 On High-Efficiency Power Amplifiers ............................................342 


\subsection{Introduction}

Analog amplifiers are usually described as small-signal circuits whose purpose is to increase the amplitude of the input signal or act as an impedance buffer between other amplifiers stages, accepting input signals over a broad range of amplitudes and frequencies. Since power amplifier stages may deliver high output power to low-impedance loads, they are significantly different from the low-power small signal amplifiers. The large-signal nature of power amplifiers requires special design considerations that may not be significant for small-signal amplifiers.

The sections below are devoted to describing amplifiers or amplification output stages that are capable of delivering high-output power levels.

\subsection{Fundamentals of Power Amplifiers and Output Stages}

The output stage of an amplifier must be able to deliver a significant amount of power into a low-impedance load, such as a stereo amplifier to a pair of speakers or a radio frequency amplifier to a broadcasting antenna, with acceptably low levels of signal distortion. They are also used as output stages in integrated circuits. Because high output powers may be involved, the efficiency of the amplifier to convert a low-power signal to high power becomes increasingly important. Inefficiency causes unwanted increases in transistor operating temperature and may lead to accelerated device failure.

As a consequence, it is usually required to have one or more of the following desirable properties:

1. Large output current or voltage swing

2. Low output impedance

3. Low standby power

In addition to these basic properties, an output stage is also required to have sufficiently good frequency response and thus it will not present a limitation on the rest of the amplifier circuit.

In this section, several output stage configurations will be examined with special regards to their advantages and limitations, starting with the simplest configurations and moving on to more complex designs. 


\subsubsection{Concepts of Efficiency and Distortion for a Power Amplifier}

\subsubsection{Efficiency in Power Amplifiers}

To compare the design of power amplifiers, different figures of merit or parameters can be utilized. One of the most used is the efficiency. Notice that efficiency gives us a way to compare two different designs because it indicates how well an amplifier converts the DC input power to AC output power. The higher the efficiency, the better the amplifier is at converting the DC to AC power. This issue is important in battery-operated equipment because high efficiency means that the batteries last longer.

In order to determine the efficiency of a power amplifier, firstly it is necessary to determine the DC power supplied by the DC power source to the amplifier, $P_{D C}$. The power supplied by a DC voltage source, $V_{C C}$, is given by:

$$
P_{D C}=V_{C C} I_{D C}
$$

where $I_{D C}$ is the current provided by the DC voltage source. On the other hand, it is necessary to consider the active power, $P_{\text {out }}$, provided to the load connected at the amplifier output terminals. Thus, the efficiency is defined by:

$$
\eta=\frac{P_{\text {out }}}{P_{D C}} 100 \%
$$

This equation says that the efficiency equals the AC output power divided by the DC input power. It is important to highlight that since all transistors and resistors (without considering the load resistor) of any power amplifier waste power, the efficiency is always lower than $100 \%$.

\subsubsection{Distortion in Power Amplifiers}

When the output signal waveform of an amplifier differs in general shape from the input signal waveform, the output is said to be distorted. In particular, if a single-frequency signal input to an amplifier results in an output signal composed of the input frequency (fundamental component) and other frequencies, the amplifier has distorted the signal. The creation of these additional frequencies, known as Fourier harmonics components, is typically the result of non-linear distortion.

Large input signal to power amplifiers causes the amplifier to yield distorted output signals. In fact, in large-signal operation, the signals overtake the limits of the transistor forward-active region, causing distortion at the output. The relative amplitude of the fundamental component decreases with respect to the Fourier component as distortion increases. 
Distortion is defined in one of several ways depending on the particular application of the circuit. However, the most important, commonly used for audio circuits, is the one named as total-harmonic distortion (THD).

If we define the input signal, $v_{i n}(t)$, to a linear power amplifier as a sinusoidal input:

$$
v_{\text {in }}(t)=A_{\text {in }} \cos \omega t
$$

where $A_{\text {in }}$ is the input amplitude, it has an ideal transfer characteristic described by:

$$
v_{\text {out }}(t)=A_{0}+A_{v} v_{\text {in }}(t)=A_{0}+A_{v} A_{\text {in }} \cos \omega t
$$

where:

$v_{\text {out }}(t)$ is the output voltage

$A_{0}$ is the DC offset voltage at output

$A_{v} \quad$ is the voltage gain.

Notice that there is not harmonic distortion since the single output frequency (apart from the DC component) matches the input component $\omega$.

However, if the real output voltage is defined as

$$
v_{\text {out }}(t)=A_{0}+A_{1} \cos \omega t+A_{2} \cos 2 \omega t+A_{3} \cos 3 \omega t+A_{4} \cos 4 \omega t+\ldots,
$$

there is harmonic distortion, and $A_{0}, A_{1}, A_{2}, A_{3}, A_{4} \ldots$ are the Fourier coefficients of the output voltage. The THD is commonly given as a percentage and is expressed as the ratio of the RMS (root-mean-square) values of all the harmonic terms to the effective value of the fundamental:

$$
\mathrm{THD}=\frac{\sqrt{A_{2}^{2}+A_{3}^{2}+A_{4}^{2}+\ldots}}{A_{1}} \cdot 100 \%
$$

As noted above, THD is used extensively in audio amplifier specifications. As an example, audio amplifiers that incorporate negative feedback to compensate for non-linearity typically have THD of less than $0.003 \%$ at low frequencies and low output power levels.

\subsubsection{Power Amplifiers Classification}

In order to classify an amplifier as a high-power amplifier, it must be capable of handling large-signal amplitudes where the current and voltage swings may be a significantly large fraction of the bias value. Then, this classification can be carried out according to the portion of the period of the output waveform during which the circuit transistors conduct. The conduction angle of each transistor in the circuit, assuming a sinusoidal input, determines the 


\section{TABLE 12.1}

Classification of Power Amplifiers and Output Stages as a Function of the Transistor Conduction Angle

\begin{tabular}{lc}
\hline Amplifier Class & Individual Transistor Conduction Angle \\
\hline A & $360^{\circ}$ \\
B & $180^{\circ}$ \\
AB & $180^{\circ}-360^{\circ}$ \\
C & $<180^{\circ}$ \\
\hline
\end{tabular}

designation of the amplifier. The amplifier classification by conduction angle for sinusoidal inputs is shown in Table 12.1. The output (collector or drain) current through a transistor for class- $\mathrm{A},-\mathrm{B},-\mathrm{AB}$, and $-\mathrm{C}$ power amplifiers is shown in Figure 12.1.

In Figure 12.1a, named as class-A operation, the current flows through the transistor over the whole period. In class-B operation, shown in Figure 12.1b, each transistor only conducts over half the period of the sinusoidal waveform. Class-AB operation, shown in Figure 12.1c, illustrates current flow through each transistor for greater than a half-cycle but less than the full cycle of the input sinusoid. Figure $12.1 \mathrm{~d}$ shows class-C operation, where each transistor conducts over less than a half-cycle of the input sinusoid.

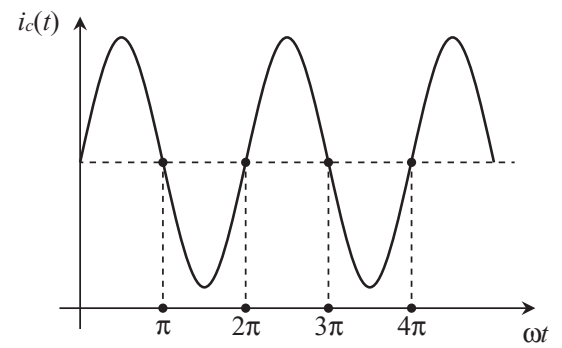

(a)

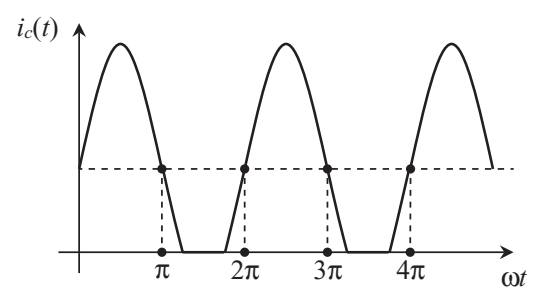

(c)

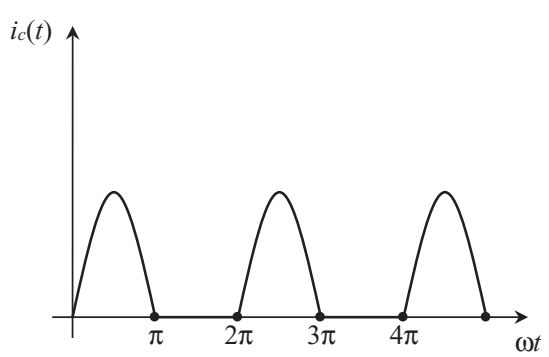

(b)

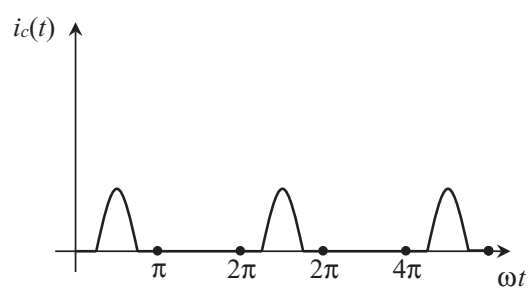

(d)

\section{FIGURE 12.1}

Transistor current for different amplifier classes: (a) Class-A amplifier, full current flows; (b) Class-B amplifier, half-period current flow; (c) Class-AB amplifier, greater-than-half-period current flows; (d) Class-C amplifier, less-than-half-period current flows. 
Class-A operation is the only configuration that will yield low distortion signals. Class-B and class-AB power amplifiers assure signal continuity by making use of arrangements of two transistors that allow each transistor to share portions of the input signal conduction angle. Class- $\mathrm{C}$ amplifiers provide single-frequency sinusoidal output by driving resonant circuits over a small portion of the cycle. The continuity of the sinusoidal output is assured by the tuned circuit. This kind of amplifier is used for narrow-band signal applications.

\subsubsection{Class-A Output Stages}

In a class-A amplifier, the output stage is biased in the active region such that there is an uninterrupted flow of current during the entire cycle, and at no time does the transistor go into its cut-off or saturation regions. As a consequence, the amplifier operates in the active region during the entire $360^{\circ}$ cycle of the input signal.

Consider the power amplifier shown in Figure 12.2 with a power supply voltage of $+V_{C C}$. The maximum peak-to-peak swing of the output voltage cannot exceed $V_{C C}$ volts. Even more, in most practical cases it will be limited to 1 or $2 V$ less than $V_{C C}$ and sometime more, in part due to distortion considerations.

Considering Equation 12.2, it is easy to demonstrate that the maximum power conversion efficiency, $\eta_{\max }$ for a class-A amplifier is given by:

$$
\eta_{\max }=\frac{P_{\text {out }, \max }}{P_{D C}}=\frac{1}{4} \quad \text { or } \quad \eta_{\max }=25 \%
$$

Therefore, of the total DC input power, a maximum of $25 \%$ can be converted to $\mathrm{AC}$ power and delivered to the load, and the rest will be dissipated as heat by the amplifier.

It is important to note that the $25 \%$ power conversion efficiency is approached only when the amplifier is driven hard enough such that the

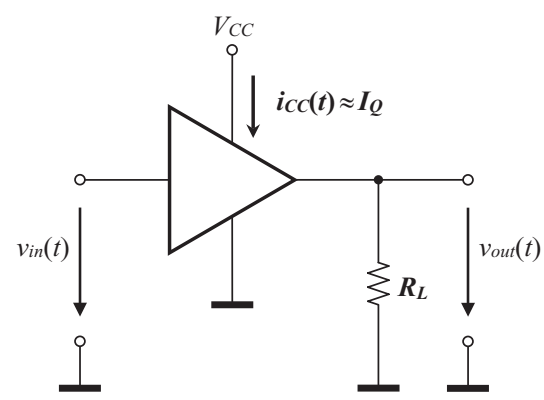

FIGURE 12.2

Class-A power amplifier. 
maximum output voltage and current swings are obtained. If the input drive conditions are different from this, the efficiency is correspondingly less. In any case, it is not really possible to achieve an efficiency of $25 \%$ in practice due, in part, to other circuit losses. To limit distortion to an acceptable level, the output swing is often limited to no more than about $50 \%$ to $80 \%$ of the total active region voltage and current spans. As a result, the actual power conversion efficiency values of class-A power amplifiers generally fall in the range of $10 \%$ to $20 \%$, and many times even lower if very low distortion is required.

Finally, it is important to highlight that this efficiency can be improved significantly using an output transformer to connect the load (transformercoupled class-A power amplifier). In this case, for the ideal amplifier the maximum efficiency is equal to $50 \%$. Nevertheless, the main disadvantage of this kind of class-A power amplifier is that the use of an output transformer in their design increases the price and the construction complexity, and involves transformer losses.

\subsubsection{Class-B Output Stages}

Practical applications of a class-B amplifier have an output stage comprised of two transistors whose outputs are combined in such a way as to reconstruct the full $360^{\circ}$-waveform cycle. Each transistor operates in the class-B mode and conducts during alternate half-cycles of the input signal so that by combining the two outputs an amplified replica of the input signal will be obtained. The two transistors of the class-B output stage are usually connected in a push-pull arrangement, a simple example of which is shown in Figure 12.3a.

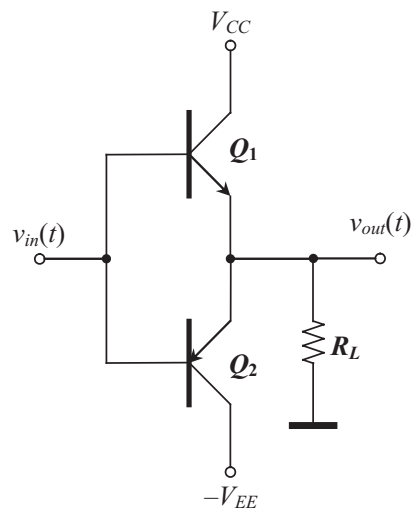

(a)

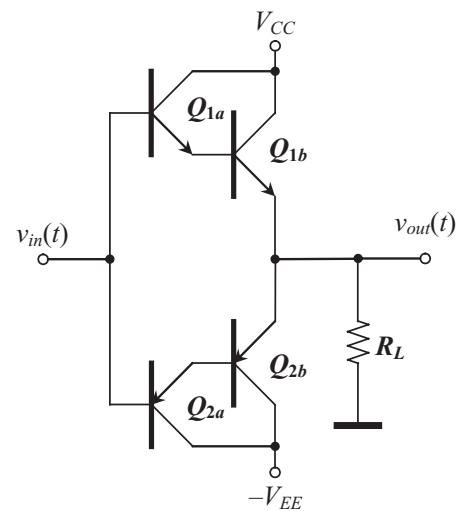

(b)

FIGURE 12.3

(a) Complementary emitter-follower class-B push-pull output stage; (b) Complementary emitter-follower class-B push-pull Darlington output stage. 
In this figure, transistors $Q_{1}$ and $Q_{2}$ constitute a complementary push-pull emitter-follower output stage operating in the class-B mode. The transistors are a complementary pair: one is an NPN transistor and the other is a PNP transistor. Both bases are fed from the same point, and when the base voltage goes in a positive direction, $Q_{1}$ will be turned on and conduct while $Q_{2}$ will be off. Conversely, when the base voltage goes negative, $Q_{1}$ will be turned off and $Q_{2}$ will be biased into conduction.

Transistor $Q_{1}$ conducts during the positive half-cycles of the input voltage applied to the $Q_{1}-Q_{2}$ output stage and will source or "push" current into the load. On the other hand, transistor $Q_{2}$ conducts during the negative halfcycles and will sink or "pull" current from the load. As a consequence, each transistor conducts for one half of the entire cycle, and the two outputs are combined to give the full-cycle $\left(360^{\circ}\right)$ sinusoidal output current. Notice that, under quiescent conditions (that is, $V_{\text {out }}=0 \mathrm{~V}$ and $I_{\text {out }}=0 \mathrm{~A}$ ), both transistors are off and the power dissipation is negligible. In the class-B mode of operation, the output transistor is biased at or very near the cutoff region, such that conduction occurs for only one half of the input waveform cycle, or $180^{\circ}$ for a sinusoidal type of input signal. In this mode of operation, the quiescent current is essentially zero and, as a result, the class-B mode of operation offers the possibility of a much higher power conversion efficiency than does the class-A mode.

For greater current gains in the output stage, $Q_{1}$ and $Q_{2}$ can be Darlington compound transistors, as shown in Figure 12.3b.

In a similar way to a class-A amplifier, it is easy to show that the maximum efficiency of a class-B output stage, $\eta_{\max }$, is given by:

$$
\eta_{\text {max }}=\frac{P_{\text {out }, \text { max }}}{P_{D C}}=\frac{\pi}{4} \approx 0.7854 \text { or } \quad \eta \approx 78.54 \%
$$

Since this is the power conversion efficiency under the conditions of maximum power output, this will be the maximum possible power conversion efficiency for a class-B amplifier. In fact, the value of $\eta$ that is actually obtained in practice is generally substantially less than this due to other circuit losses, and most of all due to the fact that the peak output voltage swing is always less than the supply voltage, typically by several volts. Part of this is due to the saturation voltage drop of the transistors, but it also results from distortion considerations. To limit distortion to an acceptable value, the output voltage swing is purposely limited to something considerably less than the full extent of the active region between cutoff and saturation.

Figure 12.4 shows a generalized graph of the power dissipation of the amplifier, $P_{d}$, versus $P_{\text {out }}$. In this plot we have two interesting points: Point ' $A$ ', when maximum output power is reached, and point ' $B$ ', when the maximum power dissipation in the amplifier transistors is achieved. Therefore, Table 12.2 shows the energy balance-sheet in ideal class-B power stages for these two points presented in Figure 12.4: In the case of maximum output 


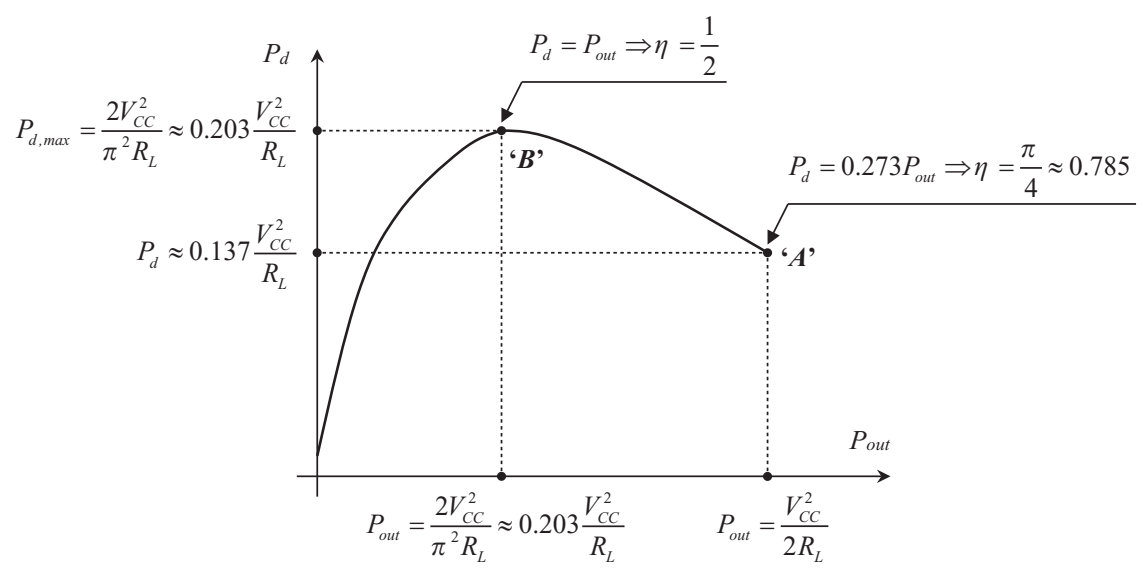

\section{FIGURE 12.4}

Power dissipation $\left(P_{d}\right)$ versus output power $\left(P_{\text {out }}\right)$ for a class-B push-pull amplifier supplied with a symmetrical power supply equal to $+V_{C C}$ and $-V_{E E}$.

power (point ' $A$ '), and in the case of maximum power dissipation in the amplifier transistors (point ' $B$ '). Notice that all expressions are given as a function of the maximum power reached on the output load.

The ratio of output power to power dissipation is limited to a maximum value of 0.33 for ideal transformerless class-A amplifiers, and unity for transformer-coupled class-A stages, compared to a ratio of 3.66 for class-B amplifiers. As a consequence, class-B amplifiers offer a substantial advantage over class-A stages. However, push-pull class-B amplifiers suffer an important type of distortion known as crossover distortion (Figure 12.5). This type of

\section{TABLE 12.2}

Energy Balance-Sheet in Ideal Class-B Power Stages for the Points ' $\mathrm{A}$ ' and 'B' Presented in Figure 12.4: In the Case of Maximum Output Power (point 'A'), and in the Case of Maximum Power Dissipation in the Amplifier Transistors (point ' $\mathrm{B}$ ')

Maximum Output Power When:

Maximum Transistors' Power Dissipation

$V_{\text {out }, \text { peak }}=V_{C C}$

When:
$V_{\text {out }, \text { peak }}=2 V_{C C} / \pi$

\begin{tabular}{|c|c|c|c|}
\hline \multirow[t]{3}{*}{$\begin{array}{l}\text { Total power that is } \\
\text { drawn from the } D C \\
\text { power supply: } \\
P_{D C}=1.274 P_{\text {out }, \max }\end{array}$} & $\begin{array}{l}\text { Load power: } \\
\qquad P_{\text {out } \max }=\frac{V_{C C}^{2}}{2 R_{L}}\end{array}$ & $\begin{array}{l}\text { Total power that is } \\
\text { drawn from the DC } \\
\text { power supply: } \\
P_{D C}=0.812 P_{\text {out } \text { max }}\end{array}$ & $\begin{array}{l}\text { Load power: } \\
P_{\text {out }}=0.406 P_{\text {out }, \text { max }}\end{array}$ \\
\hline & $\begin{array}{l}Q_{1} \text { 's power } \\
\text { dissipation: } \\
P_{d, Q 1}=0.137 P_{\text {out }, \text { max }}\end{array}$ & & $\begin{array}{l}Q_{1}{ }^{\prime} \text { s power } \\
\text { dissipation: } \\
P_{d, Q 1, \text { max }}=0.203 P_{\text {out }, \text { max }}\end{array}$ \\
\hline & $\begin{array}{l}Q_{2}^{\prime} \text { s power } \\
\text { dissipation: } \\
P_{d, \mathrm{Q} 2}=0.137 P_{\text {out } \max }\end{array}$ & & $\begin{array}{l}Q_{2}^{\prime} \text { 's power } \\
\text { dissipation: } \\
P_{d, Q 2, \max }=0.203 P_{\text {out } \max }\end{array}$ \\
\hline
\end{tabular}






\section{FIGURE 12.5}

Transfer characteristics of the complementary push-pull emitter-follower output stage with crossover distortion.

distortion occurs because a small base-to-emitter voltage is required before collector current will flow, and is due to the very low or almost non-existent gain of the transistors in the cutoff region.

Class-B power amplifiers have the following advantages compared to class-A stages:

- Class-B power amplifiers have very small no-signal power dissipation in transistors. However, class-A stages causes maximum power dissipation of the transistor under no-signal conditions.

- If transistors with the same power rating are used for class-A and class-B amplifiers, the seconds will deliver more power to the load.

- Class-B power amplifiers have higher theoretical maximum efficiency than their class-A stage.

However, class-B power amplifiers also have some important drawbacks compared to class-A stages:

- Typical class-B stages require two or more transistors (or even two center-tapped transformers).

- Class-B amplifiers require NPN and PNP transistors with reasonably similar characteristics.

- The reduction of crossover distortion in class-B power amplifiers requires additional circuitry. 
When technological limitations did not allow high-quality PNP power transistors, a balanced center-tapped input transformer, which splits the incoming waveform signal into two equal halves and which are $180^{\circ}$ out of phase with each other, could be used in order to obtain a class-B power amplifier. Another center-tapped transformer on the output is used to recombine the two signals, providing the increased power to the load. The transistors used for this type of transformer push-pull amplifier circuit are both NPN transistors with their emitter terminals connected together. Obviously, two of the main disadvantages of this kind of class-B power amplifier are that it uses balanced center-tapped transformers in its design, making it expensive and difficult to construct, and an increase of stage losses.

\subsubsection{Class-AB Output Stages}

In order to avoid crossover distortion, it is necessary to bias the transistors with a small quiescent current at a point that is slightly into the active region. This can be done by applying a small DC bias voltage between the bases of the two transistors. The total bias voltage required between the two transistor bases is in the range of $1.0-1.5 \mathrm{~V}$, approximately.

Although there are different alternatives in order to bias the transistors, Figure 12.6 shows a typical example of a simple class-AB complementary push-pull emitter-follower output stage based on bias diodes. Transistor $Q_{3}$ operates as a common-to-emitter gain stage with a current source active load of strength $I_{Q}$. The push-pull output stage is comprised

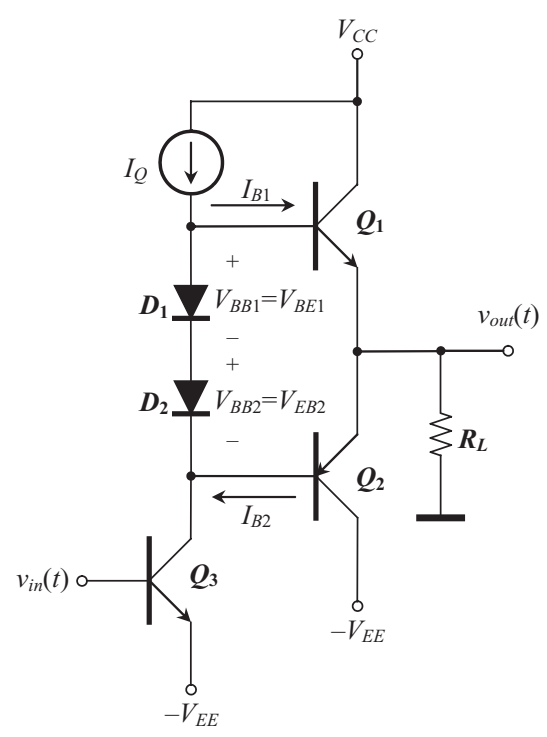

FIGURE 12.6

Class-AB push-pull output stage. 
of transistors $Q_{1}$ and $Q_{2}$. The voltage drop across diodes $D_{1}$ and $D_{2}$ is the bias voltage for a class- $\mathrm{AB}$ operation of $Q_{1}$ and $Q_{2}$. In fact, in integrated circuit applications, these two diodes will actually be diode-connected transistors. The active areas of these transistors are scaled so as to obtain the desired standby or quiescent current through the output transistors $Q_{1}$ and $Q_{2}$. The voltage drop across $D_{1}$ and $D_{2}$ decreases with temperature, but this temperature variation is exactly what is needed to compensate for the negative temperature coefficient of the base-to-emitter voltage $Q_{1}$ and $Q_{2}$, avoiding thermal runaway in these power transistors.

Since $Q_{1}$ and $Q_{2}$ are now no longer biased in the cutoff region, but slightly into the active region, the conduction angle for each transistor will be greater than $180^{\circ}$ (one half of a cycle), although it will still be considerably less than $360^{\circ}$ (a full cycle). Therefore, strictly speaking, the mode of operation is no longer class- $\mathrm{B}$, nor is it class-A. In fact, the mode of operation is called class$\mathrm{AB}$, and it combines the low distortion attribute of class-A operation with the high power conversion efficiency characteristics of class-B operation. Since the mode of operation is generally considerably closer to class- $\mathrm{B}$ than to class-A, the high power conversion efficiency available from class-B operation can still be obtained.

Along with a high power conversion efficiency, a low percentage of distortion is obtained. Indeed, with a well-balanced push-pull amplifier, the even harmonic distortion components will cancel out, leaving only the odd-numbered harmonics. Since the amplitude of the different harmonics decreases rapidly with increasing harmonic number, the cancellation of the second harmonic term, which will be the largest harmonic component, along with the other even harmonics, can lead to a very great reduction in the distortion.

For high-output power values, the circuit in Figure 12.6 can be completed substituting transistors $Q_{1}$ and $Q_{2}$ with a Darlington configuration (or by a Sziklai pair, also known as a complementary Darlington).

Finally, power amplifiers, especially those working in class-B, are usually operated under closed-loop conditions with either an internally or externally connected negative-feedback loop. The closed-loop gain, $A_{C L}$, is related to the open-loop gain, $A_{O L}$, by $A_{C L}=A_{O L} /\left(1+f \cdot A_{O L}\right)$, where $f$ is the feedback factor. Since the closed-loop gain is generally very much less than the open-loop gain, any variation in the open-loop gain results in smaller variation in the closed-loop gain. As a consequence, the amplifier gain is basically fixed by the feedback factor selected by designers and essentially independent of the characteristics of the transistors used in the amplifier implementation.

\subsubsection{Class-C Output Stages}

Class-C amplifiers are used for amplification of a single frequency (tone) or a very narrow frequency band. In class-B output stages, the output current ideally flows for exactly $180^{\circ}$ of the input sinusoidal waveform. If the duration of the output current is less than one half-cycle, class- $C$ operation takes 
place. The periodic output current generates a sinusoidal output voltage by flowing through a resonant circuit tuned to the fundamental frequency or one of the harmonic components. Therefore, with class- $\mathrm{C}$ it is always necessary to use a resonant circuit for the load. This is why almost all class-C amplifiers are tuned amplifiers. This makes them ideal for amplifying radio and television signals because each station or channel is assigned a narrow band of frequencies on both sides of a center frequency.

The DC collector current is the only current drain in a class- $C$ amplifier because it has no biasing resistors. Indeed, in a class- $C$ amplifier, most of the DC input power is converted into AC load power because the transistor and coil losses are small. For this reason, a class- $\mathrm{C}$ amplifier has high stage efficiency.

As the conduction angle of output current approaches zero degrees, the circuit efficiency approaches $100 \%$. Unfortunately, the output power also tends toward zero in this instance. Some compromise between good efficiency and high power output is necessary under normal conditions, with a resulting typical efficiency of $90 \%$.

A simple class-C amplifier is shown in Figure 12.7. The ideal input voltage, collector current, collector and output voltage waveforms are shown in Figure 12.8.

Since collector current is periodic, it will contain a fundamental component of current and higher harmonics in addition to a DC or average value. The tank circuit or parallel resonant circuit will ideally present zero impedance to DC and all harmonics except the fundamental. A resistance $R_{P}$ will be presented to the fundamental frequency. This resistance is related to the circuit $Q$ and other tuned-circuit parameters. The fundamental frequency components of current will develop an AC voltage across the tuned circuit that is in proportion to this component. The AC output voltage is given by:

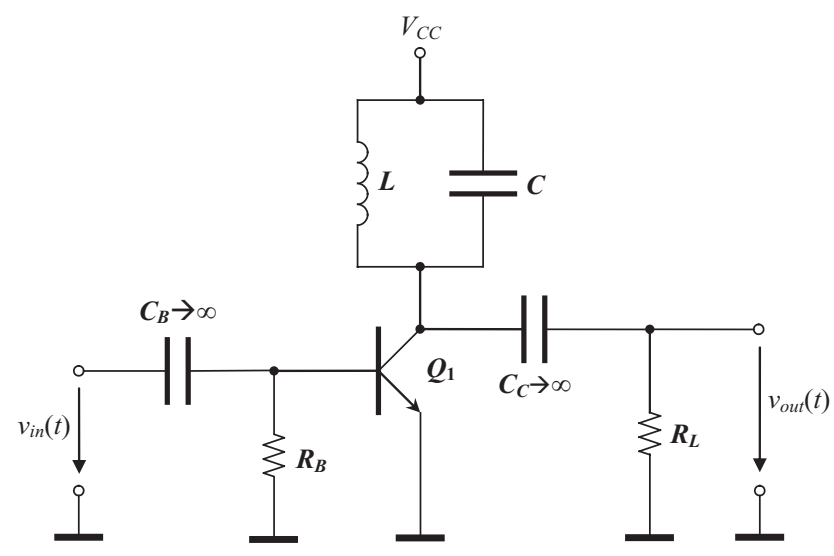

FIGURE 12.7

A basic class-C stage. 




(b)
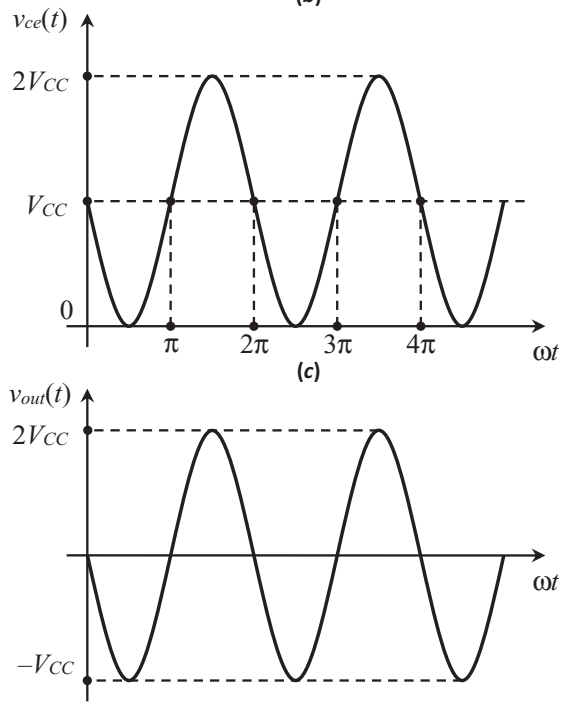

(d)

FIGURE 12.8

(a) Input voltage, (b) collector current, (c) transistor collector-to-emitter voltage, and (d) output voltage waveforms of the ideal basic class-C stage.

$$
v_{\text {out }}(t)=-R_{P} I \cos \omega t
$$

where $I$ is the magnitude of the fundamental current component. For an ideal tank circuit, there will be no distortion of the voltage waveform since load impedance exists only for the fundamental frequency. Nevertheless, in practice, the higher components may appear in the voltage waveform due to non-zero impedances presented to harmonics by the tank circuit.

Some class- $C$ stages are driven from cutoff through the active region to the saturation region. Therefore, additional distortion is introduced as the transistor output impedance drops drastically when saturated. In addition, if harmonic generation is required, the tank circuit is tuned to the desired frequency and develops a voltage only at this particular frequency. 
In order to calculate the input power, we must find the DC collector current that flows. Output power can be found only if the magnitude of the fundamental frequency component is found. For transistor stages, the collector-current waveform closely approximates a portion of a sinusoid. A Fourier analysis of this waveform yields the two quantities of interest. The circuit efficiency is given by:

$$
\eta=\frac{\frac{\alpha}{2}-\frac{\sin \alpha}{2}}{2 \sin \frac{\alpha}{2}-\alpha \cos \frac{\alpha}{2}}
$$

where $\alpha$ is the transistor's angle of conduction, being $0 \leq \alpha \leq \pi$ rad. Notice that, on the one hand, when $\alpha$ equals $\pi$, the angle of conduction is $180^{\circ}$ and the amplifier operates in the class-B mode. Therefore, the efficiency calculated from the previous equation is $78.56 \%$, which agrees with previous results obtained for a class-B amplifier. On the other hand, when the conduction angle decreases, the stage efficiency increases. Thus, an efficiency of $100 \%$ is approached as $\alpha$ tends toward zero. However, the output power also tends toward zero, resulting in a practical lower limit on $\alpha$. Figure 12.9a shows plots of efficiency as a function of $\alpha$. In applications with a variable input voltage, the output voltage will depend on both the amplitude of the input voltage and $\alpha$.

The development of maximum output power requires the output voltage magnitude to equal $V_{C C}$. When $\alpha$ decreases, the fundamental frequency component of collector current will decrease in magnitude. Thus, the resonant tank circuit impedance must be increased to develop the maximum output signal. As $\alpha$ approaches zero, a very small fundamental current component is present. Consequently, an extremely large value of $R_{P}$ is required to develop the maximum output voltage. It is reasonable to expect little output power

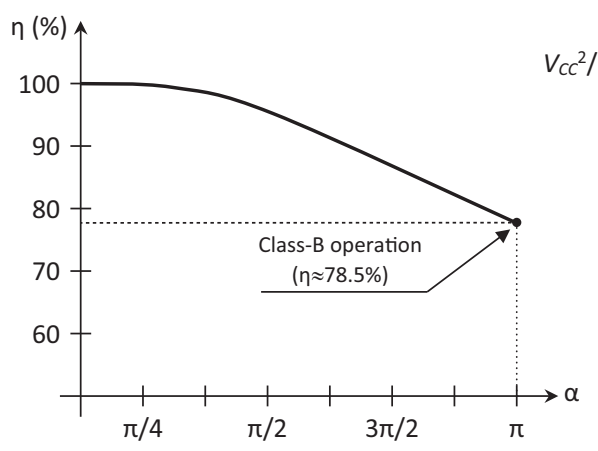

(a)

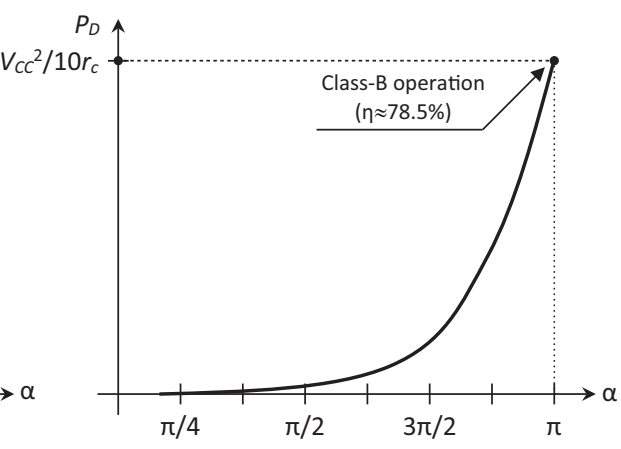

(b)

FIGURE 12.9

(a) Circuit efficiency, and (b) power dissipation of the transistor as a function of $\alpha$. 
under this condition. A reasonable compromise between circuit efficiency and output power often puts $\alpha$ in the range of $4 \pi / 6 \mathrm{rad}$ to $8 \pi / 9$, approximately, giving a conduction angle of $120^{\circ}$ to $160^{\circ}$.

The power dissipation of the transistor, $P_{d}$, depends on the conduction angle $\alpha$. As shown in Figure 12.9b, the power dissipation increases with the conduction angle up to $180^{\circ}$. The maximum power dissipation of the transistor can be derived by:

$$
P_{d}=\frac{\mathrm{MPP}^{2}}{40 r_{c}},
$$

where

MPP is the maximum output, ideally given by $2 V_{C C}$

$r_{c}$ is the AC resistance seen by the collector at resonance (approximately equal to the equivalent parallel resistance of the inductor in parallel with the output load).

This equation represents the worst case. A transistor operating as class- $\mathrm{C}$ must have a power rating greater than this or it will be destroyed. Under normal drive conditions, the conduction angle will be much less than $180^{\circ}$ and the transistor power dissipation will be less than the value given by the previous equation.

\subsection{High-Efficiency Power Amplifiers and Output Stages}

As mentioned above, linear amplifiers, such as class-A, -B or -AB, have limited efficiency because the transistors of their output stages operate in their linear region. In order to improve the efficiency of power amplifiers based on linear operation, two different technological approaches can be considered. However, the output stages obtained in both cases are classified as highefficiency power amplifiers.

This section is devoted to describing the operating principles of these amplifiers, to introduce their advantages and drawbacks, and to present some commercial application areas.

\subsubsection{Introduction and Context}

The first approach to reducing the energy losses in the power transistors used in analog output stages is to avoid the operation of these components in their linear region, thus eliminating the power losses due to devices' biasing and linear operation. As a consequence, the power transistors must operate by switching between saturation and cutoff regions, and then the power losses can be attributed basically to devices' on-resistances and switching operation. 
The high-efficiency amplifiers based on this approach are usually classified as class-D, class-E and class-F, being also referred to as switching amplifiers or digital amplifiers.

The second approach to high-efficiency amplifiers is based on linear amplifiers (power transistors operating in the linear region) using an optimized power supply system. In this regard, the voltage supply of the linear amplifier is automatically adapted in accordance with the amplifier output voltage level. If the voltage of the power supply is adequately adjusted, the power losses of the linear amplifiers due to linear operation can be greatly reduced.

High-efficiency amplifiers based on linear amplifiers with adjustable power supply are classified as class- $\mathrm{G}$ and class- $\mathrm{H}$, according to the strategy used for the voltage supply adjustment.

Because the power losses in power amplifiers are always dissipated as heat, the use of high-efficiency technologies in power amplifiers implies that the cooling requirements (typically large heat sinks or fans to blow/extract air over/from amplifier) can be greatly reduced or, in some cases, eliminated. As a result, these architectures increase the power density of power amplifiers, saving space and cost, these being two items of great importance in consumer products.

Consequently, they become an attractive solution for battery-powered applications and portable systems (hearing aids, headphones, smartphones and notebooks) where power efficiency is a key factor in extending the life of batteries.

On the other hand, high-power applications (such as audio amplifiers, servo motor drivers, push-pull amplifiers and radio frequency power amplifiers) can also take advantage of the high efficiency of these amplifiers' topologies.

\subsubsection{Switching Amplifiers: Class-D Amplifiers}

The first class-D amplifier was proposed in 1958 by R.L. Bright and G.H. Roger and it was protected under US patent reference 2,821,639 and titled "Transistor Switching Circuits", but the first commercial class-D amplifier came in 1964 from the British company Sinclair Radionics. The X-10 audio amplifier was developed by Clive Sinclair and Gordon Edge and was marketed as $10 \mathrm{~W}$, but in reality it was capable of only about $2 \mathrm{~W}$. In 1966 the replacement of the X-10 and X-20 amplifiers was launched by Sinclair Radionics as the Z12 audio amplifier. The Z12 was a reasonably successful product and, with it, the race of class-D amplifiers was started. History shows us, once again, that current solutions are based on old ideas.

The basic diagram of a closed-loop class-D amplifier is shown in Figure 12.10, where a pulse-width modulation (PWM) scheme is utilized. The PWM is achieved by comparing the error signal (the input signal in openloop amplifiers) to an internally generated triangle-wave or sawtooth signal, which acts as a sampling clock and fixes the system switching frequency. The right choice of this frequency must be a balance between increasing the 


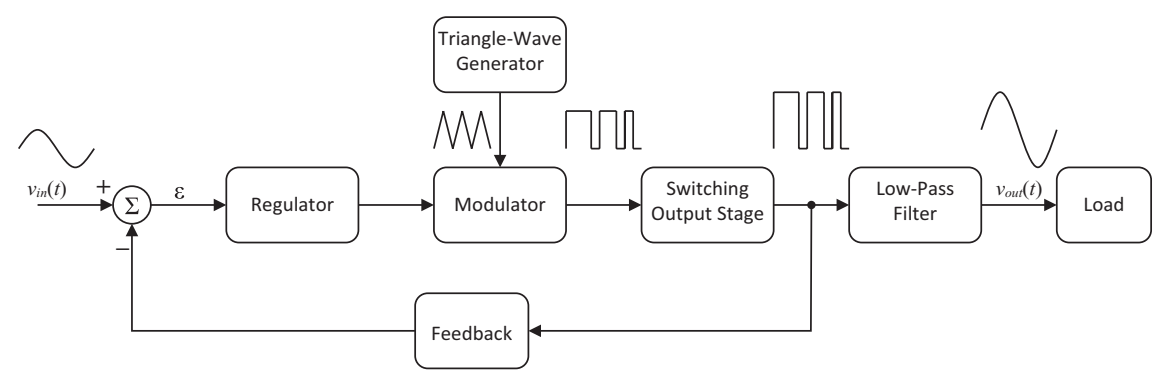

FIGURE 12.10

Closed-loop class-D amplifier block diagram.

amplifier bandwidth (BW) and decreasing the switching losses in the output stage transistors. Increasing the switching frequency increases the amplifier bandwidth, which can be at most half of the switching frequency, but switching losses will also increase, because they are proportional to the switching frequency.

The resulting duty cycle of the square- wave obtained at the modulator output is proportional to the level of the input signal. The output stage switches between the positive and negative power supplies in accordance with the PWM signal to produce a train of higher-voltage pulses. This waveform is suitable for a low-losses operation, since the output transistors have no current when they are open and have low voltage drop when they are closed, thus leading to a small amount of power dissipation. The theoretical efficiency of these amplifiers is $100 \%$ and in practice reaches values close to $98 \%$.

Finally, the PWM waveform of the output stage is fed to a lossless inductor-capacitor (LC) low-pass filter in order to extract the amplified signal and minimize electromagnetic interference (EMI) conduced to output load. If the cutoff frequency of the low-pass filter is set properly (at least an order of magnitude lower than the switching frequency), the filter output voltage is equal to the average value of the PWM voltage and so proportional to the input signal.

These amplifiers present a low power-supply rejection ratio (PSRR) because the transistors of the output stage connect the low-pass filter with the power supplies through a low resistance path. The use of a feedback loop from the low-pass filter input will improve the PSRR and also attenuate all non-low-pass filter distortions, reducing the amplifier total-harmonic distortion (THD).

For higher PSRR and lower THD operation, a feedback loop including the low-pass filter and load can be utilized. Well-designed closed-loop class$\mathrm{D}$ amplifiers can achieve PSRR values greater than $60 \mathrm{~dB}$ and THD values lower than $0.01 \%$.

Pulse-width modulation (PWM) in the most common technique used for the control of class-D amplifiers, but it is not the only one possible. In order to mitigate possible EMI emissions, alternative modulation schemes can be 
used. Some possibilities are schemes based on pulse-density modulation (PDM), random modulation technique and click modulation technique.

Circuit designs of class-D amplifiers can be subdivided into two types. Circuits which switch voltage are called voltage-mode class-D amplifiers (VMCD) and circuits which switch current are called current-mode class-D amplifiers (CMCD). The term inverse class-D, also written as class- $\mathrm{D}^{-1}$ is also used, but not widely, to refer current-switching class-D amplifiers.

A voltage-mode class-D power amplifier (VMCD) circuit consists of two active devices connected in a cascade configuration. The common junction between the devices is connected to a series output filter to reconstruct a sinusoidal load signal from the pulse train, as is shown in Figure 12.11a. In this design, the drain voltages are similar to the gates' input pulse trains (square-shape waveforms) and the current through the switches is a portion of a sine wave, so that the sum of these two currents provides a sinusoidal current through the load.

The current-mode class-D amplifier (CMCD) architecture in Figure 12.11b is fed by a DC voltage source $\left(V_{D D}\right)$ and an RF choke that has an ideal behavior similar to a DC current source. In this circuit the waveforms have been inverted if compared with the VMCD amplifier. The current through the switches is now a square-shape waveform (also similar to the gates' input pulse train) and the voltage across switches is sinusoidal. The resonant output circuit is tuned at the frequency of the switches' operation in order to achieve a sinusoidal waveform on the resistive load. This is because the higher-order harmonics are short-circuited by this parallel output network.

VMCD and CMCD amplifiers can be in low-frequency applications such as audio amplification and switch mode power supplies, but in radio frequency (RF) applications CMCD amplifiers are generally found more suitable. This is due to zero-voltage switching (ZVS) can be achieved because the voltage waveform across transistors is sinusoidal, the parasitic transistor output capacitance can become part of the resonant output filter, and the connection

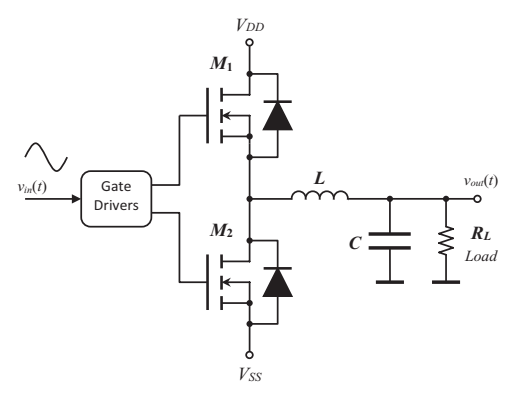

(a)

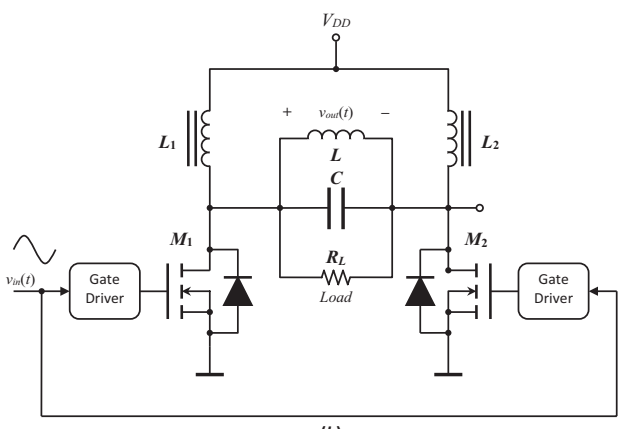

(b)

FIGURE 12.11

Class-D amplifiers: (a) Voltage-mode, and (b) current mode. 
to the circuit ground (Gnd) of the transistors' source terminals simplifies the design of gate driver circuits.

VMCD amplifiers, sometimes referred to simply as class-D amplifiers and the only ones described with some detail in this text, can be classified into two topologies according to the configuration of the output stage. These topologies are the half-bridge and the full-bridge output stage, and they can be also referred as single-ended load (SEL) and bridge-tied load (BTL) topologies respectively. Each topology has its own set of advantages and drawbacks, and they will be summarized in the following sections.

\subsubsection{Half-Bridge Output Stage}

Figure 12.11a shows the open-loop class-D amplifier topology based on a half-bridge output stage.

This topology is potentially simple when compared with topology based on a full-bridge output stage. It requires a voltage power supply system which normally uses positive and negative rails of equal magnitude $\left(V_{S S}=\right.$ $\left.-V_{D D}\right)$ and two power switches connected between them. The load is tied between the common switches point and the system ground node through a low-pass filter.

This topology can also be powered from a single supply source, with the negative supply terminal $\left(V_{S S}\right)$ used for ground. As a consequence, the output voltage swings between $V_{D D}$ and ground and remains inactive at $50 \%$ duty cycle of the PWM signal. This imposes a DC offset across the output load, equal to $V_{D D} / 2$, unless a DC-blocking capacitor is added.

In any case, the power supply system of this output stage must absorb the energy pumped back from the amplifier, especially when large reactive loads are driven. This effect, referred to as the "bus-pumping effect," results in bus voltage fluctuations which might become severe when the amplifier drives low-frequency voltages to the load. As a consequence, the output distortion increases since the gain of these amplifiers is directly proportional to the supply voltage.

\subsubsection{Full-Bridge Output Stage}

Figure 12.12 shows the open-loop class-D amplifier topology based on a fullbridge output stage.

The full-bridge topology is a more complex topology. It requires two halfbridge amplifiers with the load tied between their center points through an LC low-pass filter. However, the differential structure of the full-bridge output stage allows the utilization of three-state PWM strategies and the consequent cancellation of even-order harmonics and the possible DC offset present in the output voltage.

On the other hand, this output stage can achieve twice the output signal swing when compared to a half-bridge output stage with the same supply 


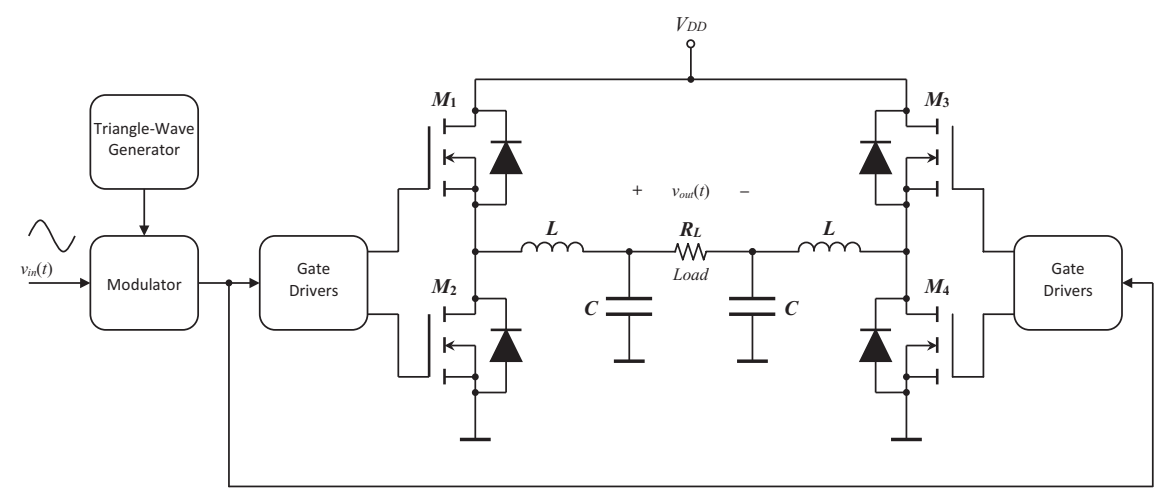

FIGURE 12.12

Output stage of bridge-tied load class-D amplifier.

voltage. This is because the load is driven in differential mode, as mentioned above. This leads to a theoretical fourfold increase in the output power when this topology is compared with a half-bridge class-D amplifier operating from the same voltage supply.

A full-bridge class-D amplifier requires twice as many transistors as a halfbridge topology. This can be considered a drawback since more switches typically mean more switching and conduction losses. This is true when high-output power amplifiers are considered, and it is due to the high output currents and supply voltages involved. Due their slight efficiency advantage, half-bridge class-D amplifiers are typically selected for high-power applications.

\subsubsection{Switching Amplifiers: Class-E Amplifiers}

The class-E amplifier concept was initially introduced by Nathan O. Sokal and Alan D. Sokal in the early 1970s and has recently received more attention due to the growing interest in high-efficiency amplifiers for transmitters in wireless communication systems.

The class-E amplifier is a single-transistor structure in switching operation with a $50 \%$ duty cycle at frequency $f_{0}$. The load network has a series resonant circuit $\left(L_{S}\right.$ and $\left.C_{S}\right)$ and a capacitor in parallel with the transistor $\left(C_{P}\right)$, as shown in Figure 12.13a.

The series resonator circuit is used to block the DC and high-frequency harmonic components, forcing the current through the load to be a sinusoid with frequency $f_{0}$. If the $V_{D D}$ choke is assumed as ideal (that is, it only conducts DC current), the current through the transistor- $C_{P}$ parallel association must then be an offset sinusoid, and this current is commutated between these two components according to the state of the switch.

If the values of reactive components are appropriately adjusted, the voltage and the derivative of the voltage in the capacitor $C_{P}$ (and transistor) can 


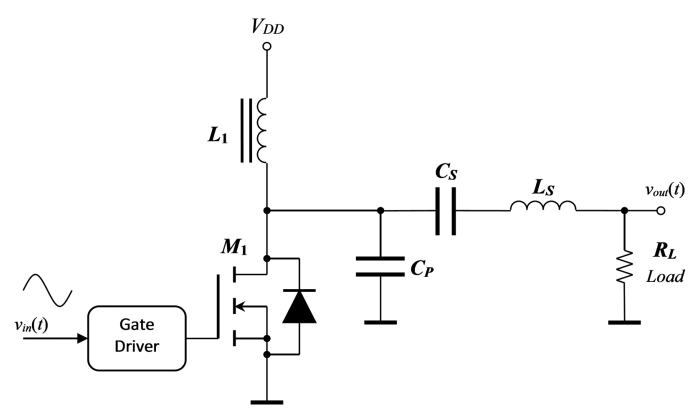

(a)

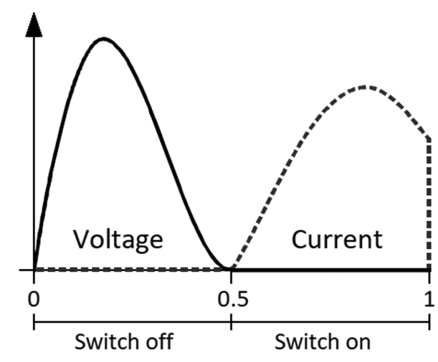

(b)

FIGURE 12.13

Class-E amplifier: (a) Structure, and (b) transistor waveforms in ZVS condition.

then be zero when the switch closes. In this condition the transistor voltage is driven to zero prior to turn-on, avoiding the overlap of voltage and current through the transistor during switching. This technique is identified as zero-voltage switching (ZVS). The transistor voltage and current waveforms for class-E amplifiers operating in ZVS conditions are presented in Figure 12.13b.

One advantage of class-E amplifiers is the easy incorporation of the transistor output capacity into the circuit topology, since $C_{P}$ is the association of all parasitic capacitances and the exterior capacitance added to the circuit to allow adjustment of resonances.

\subsubsection{Switching Amplifiers: Class-F Amplifiers}

Class-F amplifiers utilize harmonic resonators in the output networks in order to reduce losses in the transistors, thus increasing the amplifier efficiency. Their basic principles of operation were patented by Henry J. Round in 1919, and suitably formalized by V. J. Tyler in a document published by the Marconi Review in 1958 entitled "A New High-Efficiency High-Power Amplifier".

The class-F approach has been developed in order to increase the efficiency of class-AB or class-B amplifiers. It is usually based on a standard class-B amplifier wherein a parallel resonant circuit $\left(L_{0}\right.$ and $\left.C_{0}\right)$ is used to force the output voltage to be sinusoidal. Additional tuned networks are added in series with the load/resonator combination, as shown in Figure 12.14a, in order to open-circuit the transistor drain at the low-order odd harmonics. The amplifier shown in Figure 12.14a includes only two tuned networks at 3rd and 5th harmonics, but there is no theoretical limit to the number of tuned networks to be used in cascade.

By the use of these tuned networks, the transistor drain voltage waveform will begin to increasingly resemble a square wave as the generated odd harmonics will tend to flatten the top and bottom of the waveform, as can be 

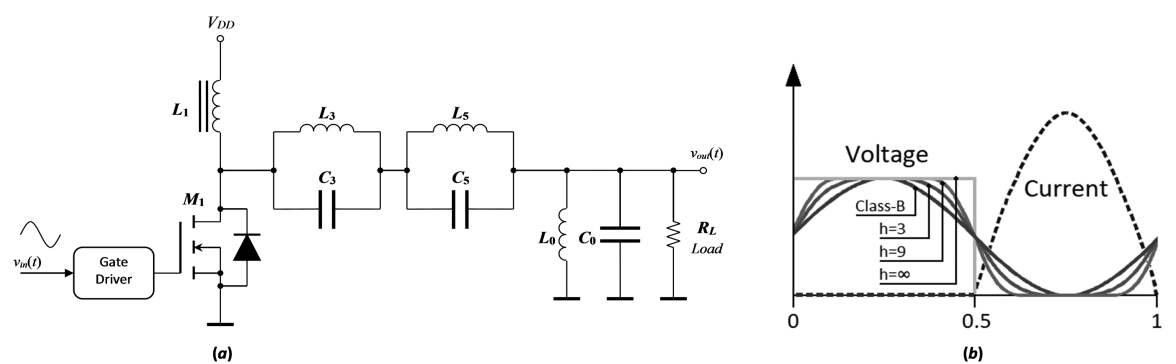

FIGURE 12.14

Class-F amplifier: (a) Structure, and (b) waveforms in the transistor drain.

seen in Figure 12.14b. This voltage shape decreases close to zero the drain voltage during the time for which the current is maximum through it and, as a consequence, the amplifier efficiency is increased. This efficiency increases quickly beyond class-B amplifiers if lower-order harmonics are tuned but the efficiency rising ratio decreases as the number of tuned harmonics increases. For instance, the maximum efficiencies for tuning up to the 3rd, 5th and 7 th harmonics will be $88 \%, 92 \%$ and $94 \%$ respectively. When the number of tuned harmonics approaches infinity, the voltage waveform is a square wave and the efficiency limit is $100 \%$, as in the class-D and class-E amplifiers.

The inverse class-F or class- $\mathrm{F}^{-1}$ amplifier is the dual tuning of a class-F one. Where class-F amplifiers short-circuit even harmonics and open-circuit odd harmonics, class- $\mathrm{F}^{-1}$ amplifiers open-circuit the tuned even harmonics and short-circuit the tuned odd harmonics. This has the effect of interchanging the transistor voltage and current waveforms. As a consequence, the voltage waveform resembles a half-sinusoid and the current waveform resembles a square wave.

As is done in this text, it is usual to classify the class-F and class- $\mathrm{F}^{-1}$ amplifiers in the switching amplifiers category since they have similar voltage and current waveforms at the transistor drain and achieve similar efficiencies. However, other authors classify these amplifiers as saturated transconductance amplifiers (or saturated amplifiers) with harmonic tuning.

\subsubsection{Switching Amplifiers Comparison}

Class-D amplifiers are mainly used in low-frequency applications; it is usual to find this topology in audio-frequency amplifiers operating with a switching frequency between $500 \mathrm{kHz}$ and $2 \mathrm{MHz}$. For operation at higher frequencies the amplifier efficiency is limited by the switching losses due to the transistor parasitic capacitance discharge in the turn-on commutation. In this regard, class-E amplifiers integrate the transistor capacitance in the topology (ZVS tuning network), allowing a higher-frequency operation without significant efficiency losses. Additionally, class-E amplifiers may be implemented with a relatively simple circuit. 
On the other hand, one of the main advantages of the class-D topology is the low voltage swing across the transistors, which is equal to the supply voltage. This is a great drawback in the case of class-E amplifiers where the voltage swing across the transistor is larger (nearly four times the supply voltage). Thus, compared with class-E topology (and also class-F) the class-D amplifier is more suitable for high-voltage applications.

Operating frequencies in class-F amplifiers are generally higher than for class-E amplifiers, but the efficiency limitations due to the lack of a simple implementation of tuned circuits for almost ideal switching conditions makes this design a poor alternative for frequencies where a class-E amplifier can be implemented.

Nevertheless, the waveforms achievable by class-F and class- $\mathrm{F}^{-1}$ amplifiers should allow performance benefits over class-E topology. In this regard, class-F and class- $\mathrm{F}^{-1}$ topologies reduce peak voltage present in the amplifier transistors (about twice the supply voltage) and, in the case of class- $\mathrm{F}^{-1}$ topology, RMS current trough amplifier transistors.

\subsubsection{Amplifiers with Adjustable Power Supply Voltage}

High-efficiency amplifiers based on switching topologies improve the overall amplifier efficiency by avoiding the overlap between voltage and current through the output stage transistors. As a consequence, power losses in the output transistors are reduced to those produced in switch-on and switchoff processes, as long as conduction losses can be neglected.

The second approach to high-efficiency amplifier topologies is based on class-AB (or class-B) amplifiers and reduces the losses in output stage transistors by adjusting the power supply voltage value in accordance with the amplifier output voltage. A reduced value of collector-emitter voltage, which is the difference between power supply voltage and amplifier output voltage, implies low conduction losses caused by wasted heat in the output stage transistors and the consequent rise of the amplifier efficiency.

The following sections are devoted to describing this second kind of highefficiency amplifiers, which usually are known as adjustable power supply amplifiers or adjustable (moving) rails amplifiers. There are two different approaches for their implementation: Class-G topology focuses on voltage rail switching, whereas class-H topology utilizes a rail-modulation strategy. Although there are multiple references to these amplifiers in the specialized literature, technically speaking neither class-G nor class- $\mathrm{H}$ amplifiers are officially recognized.

\subsubsection{Class-G Amplifiers}

As was mentioned previously, the class-G topology optimizes the amplifier power supply using, as a minimum, two different bipolar supply rails, but it is possible to find architectures based on two different strategies. The 
first one is based on switching the supply voltage of the class-AB amplifier between the available power rails, and the second one uses the rail-boosting strategy. In any case, the objective is adapting the supply voltage to the output voltage value (in order to prevent output clipping effect) using more than one power supply rail.

In 1977, Hitachi introduced a range of audio amplifiers under the denomination of Dynaharmony which was based on class-G amplifiers using the rail-boost operating principle. In these amplifiers a $100 \mathrm{~W}$ (into an $8-\Omega$ load) class-AB amplifier and two power rails of $\pm 40 \mathrm{~V}$ and $\pm 95 \mathrm{~V}$ were used. A basic structure of amplifiers based on this strategy is shown in Figure 12.15a. In this topology, two sets of power transistors operate in series connection, referred to as the inner pair $\left(Q_{1}\right.$ and $Q_{2}$, connected to the load and driven from the low voltage rails: $\left.\pm V_{L}\right)$, and the outer pair $\left(Q_{3}\right.$ and $Q_{4}$, connected to the high voltage rails: $\left.\pm V_{H}\right)$.

As long as the output voltage $\left(V_{\text {out }}\right)$ remains between the low-voltage rails, the outer pair remains in the cutoff region and the collector voltages of the inner pair remain equal to the low rails voltages $\left( \pm V_{L}\right)$. Once the output voltage is close to exceeding the low rails voltages the outer transistors boost the collector voltages, allowing the output voltage to swing up to the full high rails voltages $\left( \pm V_{H}\right)$. Figure $12.15 \mathrm{~b}$ shows a possible output voltage and the voltage at the collectors of inner transistors (the boosted voltage). During the boost voltage operation, the voltage in the inner transistors is maintained two or three volts above the output voltage, depending on the Zener voltage value of the diodes used as level-shifters.

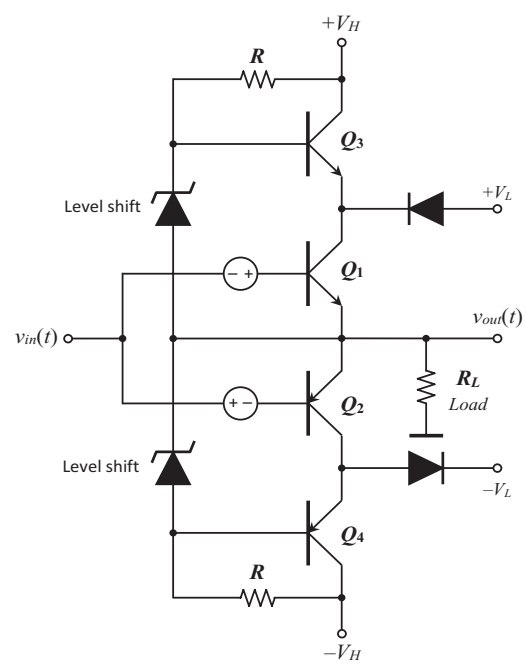

(a)

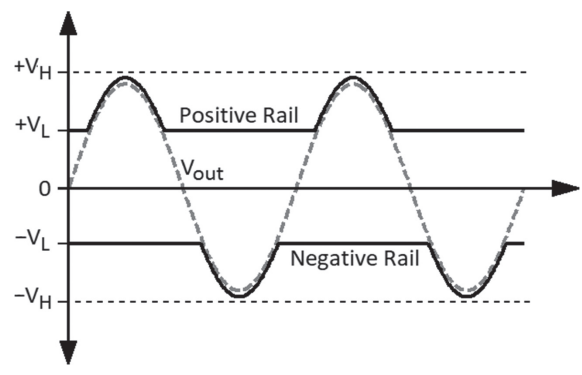

(b)

FIGURE 12.15

Class-G amplifier: (a) Boost structure, and (b) voltage rail boost effect. 


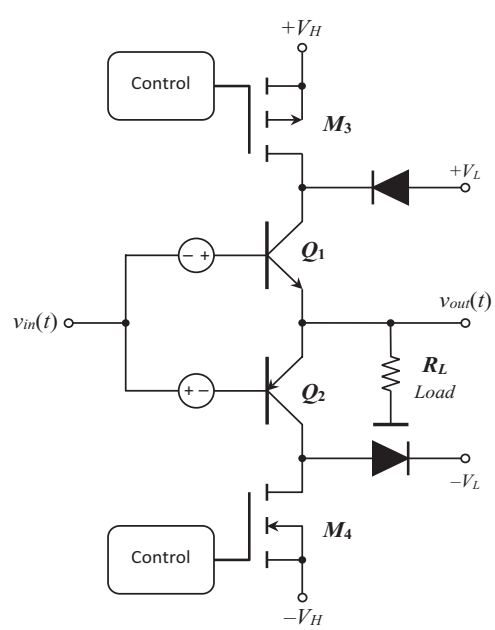

(a)

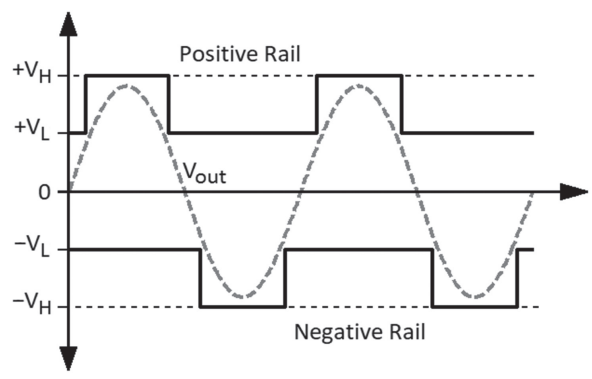

(b)

FIGURE 12.16

Class-G amplifier: (a) Switch structure, and (b) switching operation between power rails.

In 1981, Carver Corporation introduced a domestic amplifier, known as the Carver Cube, based on a refinement of Hitachi's amplifiers. This amplifier uses three bipolar power rails of $\pm 25 \mathrm{~V}, \pm 48 \mathrm{~V}$ and $\pm 80 \mathrm{~V}$ and claimed $500 \mathrm{~W}$ output when driven with music signals. The operation of this amplifier was based on switching between the three available power rails. Figure 12.16a shows a basic structure of one amplifier based on this strategy but using only two bipolar power rails. In this structure the inner pair of transistors $\left(Q_{1}\right.$ and $\left.Q_{2}\right)$ operate as a normal class-AB amplifier and the outer pair $\left(M_{3}\right.$ and $M_{4}$ ) operate as switches, allowing the modification of the collector voltages applied to the inner pair.

This amplifier operates from the lower supply voltages $\left( \pm V_{L}\right)$ until output headroom (difference between output voltage and power supply voltage) becomes an issue. At this point the control system switches the power supply of the output stage to the higher supply rails $\left( \pm V_{H}\right)$ in order to avoid the imminent output voltage clipping. When the output voltage drops below a prefixed level, the control system switches back to the lower rails $\left( \pm V_{L}\right)$. This operative is shown in Figure 12.16b. In this regard, there are several methods to control the switching operation between the power rails but the feedback from the output voltage $\left(V_{\text {out }}\right)$ or the input voltage $\left(V_{i n}\right)$, once pre-amplified, are normally used.

The design of any of these class-G amplifier topologies must achieve some compromise between circuit complexity and efficiency improvement. Two different power voltages minimizes the complexity of the power supply design, maintaining a reasonably high efficiency if the voltage values are properly selected. Additional rails may reduce power losses but increase 
the power supply complexity and reduce the overall system reliability. Amplifiers with two or three power rails have been reported in the literature, but not those using four or more rails, two-rail amplifiers being the most frequently used.

In this regard, most professional class-G audio power amplifiers use dualvoltage supply topology where the low-voltage supply is selected between $40 \%$ and $50 \%$ of the high-voltage value, but there is no optimum percentage because there are too many variables to be considered: Type of input signal, amplifier usage, power level demand and type of load. As a consequence, class-G amplifiers' efficiency depends largely on the same factors. With a high-amplitude sine wave as input signal there is no efficiency improvement compared to class- $\mathrm{AB}$ amplifiers operating under similar conditions. If the amplitude of the input signal remains at a level where the class-G amplifier operates from the low-voltage rails, then power efficiency does increase compared with class- $\mathrm{AB}$ architecture, which can only operate from the highvoltage level.

In any case, when the two operating strategies of class-G amplifiers are compared, the efficiency in the switch structures is potentially slightly higher than in the boosted structures, because the outer transistors operate in switching rather than linear mode. Consequently, the outer transistor losses are very small, but the peak dissipation of the inner transistors is increased. Nevertheless, the electric noise due to supply-rail commutation in switched structures can be translated to audible output glitches.

\subsubsection{Class-H Amplifiers}

Class-H amplifiers have a similar structure to class- $\mathrm{G}$ amplifiers and it can be difficult to decide into which category some structures of amplifier should be classified. Class- $\mathrm{H}$ is often described as topology that uses an externally modulated power supply. In other words, these amplifiers use only one power supply whose voltage value is modulated in accordance with the amplifier output voltage value to ensure reasonable headroom and thus avoid the output voltage clipping. Figure 12.17a shows the basic structure of a class- $\mathrm{H}$ amplifier which is based on a class- $\mathrm{AB}$ amplifier and an adjustable power supply system.

It is possible to find in the specialized literature two different strategies for generating the supply voltages of these amplifiers. The first one uses a buck or boost DC/DC converter as power system to generate two different voltage values from a single external supply voltage. The operation is similar to that in class-G switch structure shown in Figure 12.16b. This strategy is used by the company Maxim Integrated in their integrated headphone amplifiers.

The second strategy also utilizes a DC/DC converter as a tracking power supply system which monitors the amplifier output voltage and adjusts the supply voltage accordingly. When the amplifier output voltage increases above a threshold value, the power supply system tracks the peak level of the 


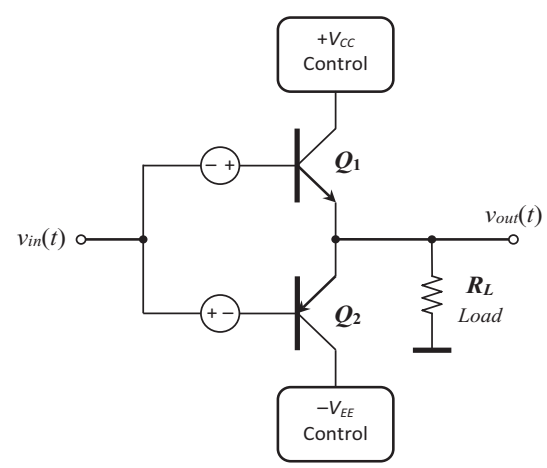

(a)

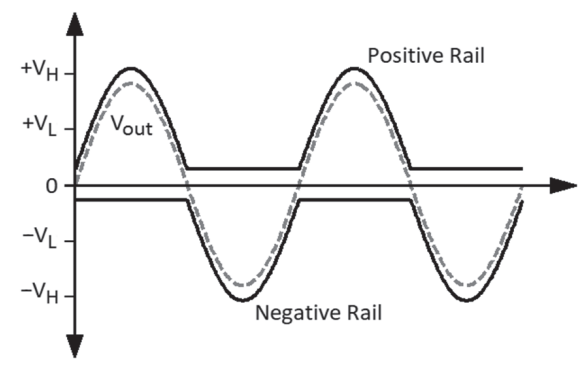

(b)

FIGURE 12.17

Class-H amplifier: (a) Structure and (b) voltage of power rails.

output voltage and impose a supply voltage only slightly higher $(\Delta V)$ than the instantaneous value of the output voltage, as is shown in Figure 12.17b. When the amplifier output falls below the threshold value, the power supply system returns to its nominal output voltage value. As a consequence, power dissipation is greatly reduced compared to conventional class-AB amplifiers. This second strategy is used by the company Texas Instruments in their integrated amplifiers for piezoelectric actuators and ceramic speakers. These amplifiers are also referred to by some authors as amplifiers with tracking power supply, and their main operating principle is used in envelope-tracking amplifiers devoted to RF applications.

When class-G and class-H amplifiers are compared, the need for multiple supplies used in class-G output stages may be a problem. The use of a multiple tap transformer is a good solution in amplifiers powered from the mains, but in the case of portable systems fed from batteries, it is an important drawback. On the other hand, the use of DC/DC converters as power supply in class- $\mathrm{H}$ amplifiers implies an inevitable ripple in the supply voltage. This perturbation in the power supply voltage is minimized by the voltage headroom utilized $(\Delta V)$ in the power supply system and also reduced by the PSRR of the class$\mathrm{AB}$ stage. However, it inevitably affects the amplifier quality by increasing the $\mathrm{THD}$ value. Therefore, class- $\mathrm{H}$ offers a similar quality to class- $\mathrm{AB}$ amplifiers with efficiency that more or less matches the efficiency of class-D topologies.

\subsubsection{Other High-Efficiency Power Amplifier Topologies}

It should be mentioned that, in addition to the major classes of power amplifiers that were treated in previous sections, there are other documented structures of high-efficiency power amplifiers, usually based on a combination or association of stages used in the major classes. Therefore, in the remainder of this section they are briefly considered. 


\subsubsection{Class-FE and class-E/F Amplifiers}

Class-FE and Class-E/F were recently introduced (the first patent dates from 2000) as power amplifier topologies which incorporate the best performance aspects of class-E and class-F or class- $\mathrm{F}^{-1}$ topologies, avoiding their main drawbacks but increasing the complexity of the amplifier design.

Zero voltage and zero voltage-derivative conditions (soft-switching operation) corresponding to the class-E amplifier can be used to eliminate discharge loss of the transistor capacitance, and harmonic tuning can be provided by using resonant circuits tuned to selected harmonic components, realizing class-F or class- $\mathrm{F}^{-1}$ mode with improved collector waveforms.

Class-FE and class-E/F amplifiers incorporate the transistor output capacitance into the tuned circuit, as in the class-E topology, and also minimize the peak voltage, as in the class- $\mathrm{F}$ and class- $\mathrm{F}^{-1}$ topologies. Additionally, class$\mathrm{E} / \mathrm{F}$ amplifiers minimize the RMS current trough in the amplifier transistors, as occurs in the class- $\mathrm{F}^{-1}$ topology.

\subsubsection{Class-DE Amplifiers}

The class-DE amplifier topology (first proposed in 1975) is one of the optimized voltage-mode class-D (VMCD) topologies, where both of two transistors satisfy class-E switching conditions. Class-E switching conditions mean that both the switching voltage and the derivative of switching voltage are zero when each transistor turns on. This functionality is achieved by the addition of a shunt capacitor to each transistor and the addition of two deadtime intervals on the transistors' driving pattern, when both transistors are switched off. Since the shunt capacitors must be discharged at that exact time, an additional series inductor with optimum value must be included in the load network.

Achieving the class-E switching conditions in the output stage allows class-DE amplifiers to operate with a very high power-conversion efficiency at high operating frequencies (MHz-order operation).

\subsubsection{Class-AB/D Amplifiers}

As mentioned above, the main problems of class-D amplifiers are related to the switching ripple at the output and the inherent difficulty of achieving a low output distortion. Class-AB amplifiers do not suffer from these problems, but they have a low efficiency. There are two basic topologies that allow the combination of these two amplifiers: By connecting them in series or in parallel.

In the series connection, the class-D amplifier generates the supply voltage for the class-AB amplifier. This topology is usually referred to as a class- $\mathrm{H}$ amplifier and it was previously described in Section 12.3.6.2.

On the other hand, the main goal of connecting linear (class-AB) and switching (class-D) amplifiers in parallel is to maximize the part of the 
output current provided by the efficient class-D amplifier and to minimize the output current of the class- $\mathrm{AB}$ amplifier. The class- $\mathrm{AB}$ amplifier operates as voltage source and determines the output voltage while the class-D amplifier operates as a current source, usually based on a half-bridge switch with a coil in series to the output.

In this configuration, the linear part controls the output voltage, while most of the output current is provided by the switching amplifier. In consequence, the power dissipation is minimized, when compared with class- $A B$ amplifiers, and the output distortion is also reduced, when compared with class-D amplifiers.

\subsubsection{Class-DG Amplifiers}

Class-DG amplifiers are based on a proprietary Maxim Integrated output stage. As the company claims in the datasheets of its products, they offer higher efficiency over a greater output power range than previous amplifier topologies. These amplifiers combine class-D switching output efficiency and class-G supply-level shifting with a multilevel output modulation scheme.

Their operating principle is simple; the output stage uses PWM, a rail-torail output signal with variable duty cycle, to generate the output voltage as in a class-D amplifier. The magnitude of the output voltage is sensed and the supply rails are switched as needed to more efficiently supply the required power. For a low-output voltage swing requirement (below the external supply rail $\left.V_{D D}\right)$, the output range is between $V_{D D}$ and ground. When output voltage swing above $V_{D D}$ is required, an internal inverting charge-pump circuit generates negative rail $\left(V_{S S}\right)$, replacing ground as the lower supply. The high-output voltage swing range is then $V_{D D}$ to $V_{S S}$, approximately double the low swing range. This approach efficiently manages power consumption by switching the operating rails as needed according to the output voltage swing requirements.

\subsection{Comparison and Results}

This chapter concludes with some results in order to present a global comparison between power amplifiers or output stages, including the most important conclusions concerning the different classes presented in this chapter. It is important to highlight that a global comparison between power amplifiers is not an easy task for commercial equipment. Considering only the comparison for audio amplifiers, there are hundreds of products and manufacturers on the world market. However, comparing audio amplifiers with other kinds, such as radio frequency amplifiers, WiFi or Bluetooth amplifiers could be inappropriate, considering the lack of rigour due to the enormous 
differences between all of these sorts of equipment in terms of characteristics and performance (for instance, power level, spectral frequency, point of load like antennas, loudspeakers, etc.). Unfortunately, the information provided by different distributors and manufacturers of audio amplifiers is not always homogeneous. Therefore, for comparison purposes, it is necessary to define a merit marker or figure to make a comparison between different classes of technology in power amplifiers. What is more, the comparison must be carried out in the same conditions, and only the case of output power over a $4 \Omega$ load has been considered.

Generally, apart from amplifier output power, information concerning dimensions and weight of the equipment is included on the specifications. Thus, an interesting and commonly used merit figure in specialized bibliography is output power with respect to the weight of the amplifier (expressed in $\mathrm{W} / \mathrm{kg}$ ).

However, the volume of an electronic device or equipment is important too, the compactness of the amplifier being an important target in our "electronic world". Therefore, the compactness of the amplifier (expressed as (volume-weight) ${ }^{-1}$ ) can be defined as an idea of how "integrated" the equipment is. In this case, on the one hand, a low value assumes a heavy and bulky amplifier, and, on the other hand, a high value corresponds to a light and a small amplifier. Obviously, the compactness is a target in the design of the equipment, and depends on the manufacturer's skill, but is mainly fixed by the technology or class of the amplifier. In addition, this consideration may be conditioned by the use of normalized rack units in professional equipment.

More than 40 amplifiers of different manufacturers (including Skytone, Altair, Focal, Extron Electronics, Beilarly Audio, SP Audio, Yamaha, Peavy, RCF, Earthquake Sound, Xibon, Samson, BKL, Electrocompaniet, D.A.S., MTX AUDIO) have been evaluated. The results obtained are presented in the next figures.

Figure 12.18 shows the power/weight merit figure (expressed in $\mathrm{W} / \mathrm{kg}$ ) with respect to power (in W). Marks in this figure represent several commercialized amplifiers on the market. Only one element is presented from class-A (cross plot mark), most being from class-AB (square plot mark), class$\mathrm{H}$ (triangle-up plot mark) and class-D (diamond plot mark) power amplifiers. The lines presented in the figure are the interpolation of the represented marks. This figure shows that class-D amplifiers (solid line) present the better ratio of this merit figure; secondly, we have class-H stages (dash-dot line), and, finally, class-AB amplifiers (dotted line). This conclusion obtained from commercial information is in accordance with the analysis presented in previous sections of this chapter.

Figure 12.19 shows a distribution of compactness (expressed in $\left.\left(\mathrm{m}^{3} \cdot \mathrm{kg}\right)^{-1}\right)$ related to power (in W). Marks in the figure correspond to several commercial audio amplifiers, in a similar way to Figure 12.18. As can be seen, the regular tendency (interpolation lines on the figure) for all the analyzed equipment 


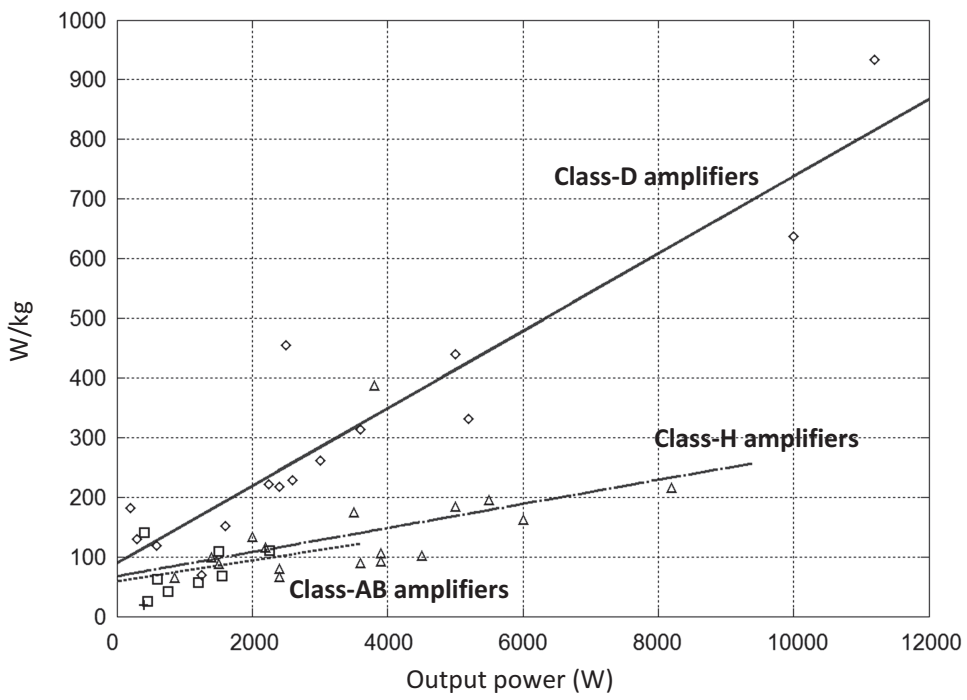

FIGURE 12.18

Power/weight indicator with respect to output power.



FIGURE 12.19

Compactness indicator with respect to output power. 
shows higher values of compactness for class-D stages (solid line). The difference with respect to the next in order (class- $\mathrm{H}$, in dash-dot line) is around five times. This difference is approximately the same as that obtained when we compare class- $\mathrm{H}$ and class-AB (dotted line).

Considering these two merit figures, we can conclude that the natural tendency of manufacturers will be to focus on the development of class-D power amplifiers and stages. Their advantages concerning weight and dimensions with respect to older technologies such as class-A and class-AB, guaranteeing excellent performance as regards "sound quality", will persuade consumers to use them and ensure their subsequent expansion in the market. In the opinion of the authors, class-H amplifiers will continue to be an excellent alternative in the market for low and medium power levels, to provide certain specialized customers with classic linear amplification and a good compromise in terms of weight and volume of equipment.

\subsection{Would You like to Learn More?}

As the reader can imagine, this section only overviews briefly some alternatives to classical high-efficiency amplifier topologies. In this sense, it is possible to find in the specialized literature more topologies of amplifiers with special relevance in RF applications or in audio systems. More information about these topologies can be found in the following references:

\subsubsection{On Fundamentals of Power Amplifiers and Output Stages}

- Active and Non-Linear Electronics. Thomas Schubert, Jr. and Ernest Kim. John Wiley \& Sons, Inc. 1996. ISBN: 0-471-57942-4.

In chapter 7 ("Power Amplifiers and Output Stages") this book discusses design principles of class-A, class-B, and class-AB power amplifiers and output stages.

- Advanced Electronic Circuit Design. David Comer and Donald Comer. John Wiley \& Sons, Inc. 2003. ISBN: 0-471-22828-1.

In chapter 2 ("Fundamental Power Amplifier Stages") and chapter 3 ("Advanced Power Amplification"), this book reviews and analyzes in depth power amplifier design principles and focuses on linear power amplifiers, especially transformer-coupled and transformerless class- $\mathrm{A}$ stages, class- $\mathrm{B}$ and class- $\mathrm{AB}$ transformer stages, transformerless class- $\mathrm{B}$ and class- $\mathrm{AB}$, class- $\mathrm{C}$ and class-D output amplifiers.

- Design and Applications of Analog Integrated Circuits. Sidney Soclof. Prentice-Hall International, Inc. 1991. ISBN: 0-13-033168-6. 
In chapter 12 ("Power Amplifiers") this book discusses in depth the design of power amplifiers, especially those devoted to microelectronic implementation in integrated circuits (especially class-A, class-B, and class-AB).

- Electronic Circuits. Electronic Circuits: Analysis, Simulation, and Design. Norbert R. Malik. Prentice-Hall International, Inc. 1995. ISBN: 978-0023749100.

In chapter 10 ("Power Circuits and Systems") this book carries out an introduction to class- $\mathrm{A}$, class- $\mathrm{B}$, class- $\mathrm{AB}$ and class-D power amplifiers.

- Electronic Principles. Albert Paul Malvino and David J. Bates. 8th ed. McGraw-Hill Education, Inc. 2016. ISBN: 978-0-07-337388-1.

In chapter 10 ("Power Amplifiers") this book carries out an introduction to class-A, class-B, and class-AB power amplifiers. In addition, the authors present an interesting analysis in this chapter of class-C power amplifiers.

\subsubsection{On High-Efficiency Power Amplifiers}

- Switchmode RF Power Amplifiers. Andrei Grebennikov and Nathan O. Sokal. Elsevier Inc. 2007. ISBN: 978-0-7506-7962-6.

This book reviews the design principles of power amplifiers and focuses on switching mode power amplifiers (class-D, class-E and class-F). Chapter 8, titled "Alternative and Mixed-Mode HighEfficiency Power Amplifiers," explains the class-DE, class-E/F, class$\mathrm{E}_{\mathrm{M}}$ and class- $\mathrm{E}^{-1}$ topologies.

- Switchmode RF and Microwave Power Amplifiers. Andrei Grebennikov, Nathan O. Sokal and Marc Franco. Elsevier Inc. 2012. ISBN: 978-0-12-415907-5.

As the previous one, this book reviews the design principles of power amplifiers and focuses on switching mode power amplifiers. Chapter 9, also titled "Alternative and Mixed-Mode High-Efficiency Power Amplifiers," includes an overview of the class-EF amplifiers and the amplifiers known as outphasing power amplifiers.

- High Efficiency Audio Power Amplifiers; Design and Practical Use. Ronan A. R. van der Zee. Universiteit Twente. 1999. ISBN: 90-36512875.

This $\mathrm{PhD}$ thesis reviews the measurement and prediction of amplifiers' dissipation, and the topologies of linear and switching amplifiers including class- $\mathrm{AB}$, class-G, class- $\mathrm{H}$ and class-D amplifiers. It also focuses on series and parallel combinations of linear 
and switching amplifiers, which are usually known as class-AB/D amplifiers.

- Audio amplifiers, class-T, class-W, class-I, class-TD and class-BS. Paul Rako.EDNNetwork. 2009.Published online:https://www.edn.com/ electronics-blogs / anablog / 4309722 / Audio-amplifiers-classT-class-W-class-I-class-TD-and-class-BS.

The author of this technical note comments that in the last few years several companies have been inventing amplifier classes, not as a legitimate architecture class, but as a marketing trick. This document lists and links these amplifiers to the different companies which produce them.

Finally, the datasheets provided by amplifier manufacturers are another source of information about these amplifier classes that are not officially recognized. This is the case for class-DG topology, produced by Maxim Integrated and described in detail in the datasheet of any of their integrated amplifiers based on this output stage (MAX98307 and MAX98308). 
